\title{
On the Second Virial Coefficient
}

\author{
M. L. Goldberger \\ Palmer Physical Laboratory, Princeton University, Princeton, New Jersey \\ (Received February 9, 1959)
}

\begin{abstract}
The second virial coefficient is calculated by a new method which exploits certain results known from formal scattering theory. In particular it is shown that the essential quantity may be expressed as the determinant of the $S$ matrix at a given energy. The present approach suggests several approximation techniques and also seems applicable to many-body problems.
\end{abstract}

$\mathrm{T}$ THERE is not the slightest doubt in anyone's mind as to how the second virial coefficient of a gas is calculated in quantum theory. ${ }^{1}$ The only excuse for giving yet another calculation is that the method is a powerful one and may perhaps be of use in other problems.

What must be evaluated is the difference between the partition function for a two-particle system with and without interaction. We write this as

$$
\boldsymbol{z}=Z(\beta)-Z_{0}(\beta) \equiv \sum_{n} e^{-\beta E_{n}}-\sum_{n} e^{-\beta \epsilon_{n}},
$$

where $E_{n}$ and $\epsilon_{n}$ are, respectively, the eigenvalues of the Hamiltonian with and without the interaction between the particles. Let us agree to ignore the trivial contribution from the center-of-mass motion and thus regard $E_{n}$ and $\epsilon_{n}$ as energies of the relative motion. We rewrite Eq. (1) as follows:

$$
\begin{array}{r}
z=\frac{1}{2 \pi i} \oint_{c} d \zeta e^{-\beta \zeta}\left[\sum_{n} \frac{1}{\zeta-E_{n}}-\frac{1}{\zeta-\epsilon_{n}}\right] \\
=\frac{1}{2 \pi i} \oint_{c} d \zeta e^{-\beta \zeta} \frac{d}{d \zeta}\left[\sum_{n} \ln \left(\zeta-E_{n}\right)\right. \\
\left.-\quad \sum_{n} \ln \left(\zeta-\epsilon_{n}\right)\right] \\
=\frac{1}{2 \pi i} \oint_{c} d \zeta e^{-\beta \zeta} \frac{d}{d \zeta} \ln \frac{\prod_{n}\left(\zeta-E_{n}\right)}{\prod_{n}\left(\zeta-\epsilon_{n}\right)}
\end{array}
$$

where the contour $C$ extends from $+\infty$ above the real axis beyond the least of the eigenvalues $E_{n}$, $\epsilon_{n}$ and returns to $+\infty$ below the real axis. ${ }^{2}$

Next we remark that $\pi_{n}\left(\zeta-E_{n}\right)$ is just the secular equation for the determination of the eigenvalues of the Schroedinger equation. It may, therefore, be written quite generally as $\operatorname{det}(\zeta-H)$ where $H=$ $H_{0}+V$ is the full Hamiltonian and $\operatorname{det} A$ means the determinant of the operator $A$. Similarly $\pi_{n}\left(\zeta-\epsilon_{n}\right)=\operatorname{det}\left(\zeta-H_{0}\right)$. In order that these

${ }_{1}^{1}$ See, for example, D. ter Haar, Elements of Statistical Mechanics (Reinhart and Company, Inc., New York, 1954), p. 193. equations have meaning we must imagine that the system is enclosed in a box so that there are a finite number of discrete levels. The passage to the limit of infinite volume is entirely painless in the method to be developed here.

We are led evidently to the study of a function $D(\zeta)$ defined by

$D(\zeta)=\frac{\operatorname{det}(\zeta-H)}{\operatorname{det}\left(\zeta-H_{0}\right)}=\operatorname{det}\left(1-\frac{1}{\zeta-H_{0}} V\right)$

It is perhaps worth remarking that although $D(\zeta)$ does not actually exist because of the singular nature of the operator $\left(\zeta-H_{0}\right)^{-1} V$ in the limit of a large volume and a continuous spectrum, our formal manipulations of it will lead to no error. As a matter of fact, only the imaginary part of In $D(\zeta=E+i \eta) \quad(\eta \rightarrow 0)$ will be required for our purposes, and this does exist. For simplicity let us assume that the potential $V$ is spherically symmetric. We may discuss then each angular momentum separately and write $D(\zeta)=\Pi\left[D_{l}(\zeta)\right]^{2 l+1}$, since each energy is evidently $(2 l+1)$ degenerate.

The properties of $D(\zeta)$ are well known and are discussed in detail by Schwinger, ${ }^{3}$ DeWitt, ${ }^{4}$ and Baker. ${ }^{5}$ For ease of reference we derive in a very crude way the results of these authors which we need. The treatment of DeWitt is the most convenient for our purposes. We note first from Eq. (2) that we will ultimately require $D_{l}(\zeta)$ for $\zeta=E \pm i \eta$, i.e., infinitesimally near the real energy axis, so we confine our attention to $D_{l}(E+i \eta)=D_{1_{+}}(E)$;

2 This method was used by G. Wentzel [Helv. Phys. Acta $15,111(1942)$ ] in field theory. It is perhaps worth noting that the solution to the problem treated by Wentzel, just as in the calculation of virial coefficients, is most easily obtained by evaluating the change in the density of states produced by the interaction (see reference 1). Some techniques closely related to ones used in our work have been used in statistical mechanics problems by K. M. Watson [Phys. Rev. 103, 489 (1956)].

${ }^{3}$ J. Schwinger, Phys. Rev. 94, 1362 (1954).

- B. S. DeWitt, Phys. Rev. 103, 1565 (1956). Much of what we shall describe is completely covered in DeWitt's paper and is included here only for the benefit of those who are not experts in formal scattering theory.

3 M. Baker, Ann. Phys. (N. X.) 4, 271 (1958). 
clearly $D_{l}(E-i \eta)=\left[D_{l_{+}}(E)\right]^{*}$. It follows from the fact that the number of energy levels of $H$ and $H_{0}$ are the same that $D_{l+}( \pm \infty)=1$ and that as $V \rightarrow 0, D_{l_{+}} \rightarrow 1$. Further, as $E$ increases from minus infinity, where the phase of $D_{l^{+}}(E)$ is zero, when it crosses over the first bound state of $H$, the phase decreases by $\pi$; if there are bound states of $H_{0}$ (which we assume are absent) the phase increases by $\pi$. Evidently this phase decrease of $\pi$ happens at each bound state and if these occur for energies $E_{B}{ }^{(l)}(<0)$, then we have

$\operatorname{Im} \ln D_{l+}(E)=-\sum_{B} \pi \theta\left(E-E_{B}{ }^{(l)}\right) \theta(-E)$

$$
\text { - } \theta(E) f_{l}(E),
$$

where $f_{l}(E)$ is yet to be determined, and $\theta(x)$ is the step function, unity for $x>0$, and zero for $x<0$.

To determine $f_{l}(E)$ we go back to the original expression for $D$ [see Eq. (2)]. We have then for $\dot{E}>0$ (writing $E_{+}=E+i \eta$, and $E_{n l}=\epsilon_{n l}+$ $\left.\Delta E_{n l}\right)$,

$$
\begin{aligned}
-f_{l}(E)=\operatorname{Im} \ln & \frac{\prod_{n}\left(E_{+}-E_{n l}\right)}{\prod_{n}\left(E_{+}-\epsilon_{n l}\right)} \\
& =\operatorname{Im} \ln \prod_{n}\left(1-\frac{\Delta E_{n l}}{E_{+}-\epsilon_{n l}}\right) \\
& \cong-\operatorname{Im} \sum_{n} \frac{\Delta E_{n l}}{E_{+}-\epsilon_{n l}} .
\end{aligned}
$$

Next we must relate the energy shift $E_{n i}$ of the $n$th level to the spacing of the unperturbed spectrum. This is done by remarking that in the absence of interaction demanding that the radial wave function of the relative motion vanish on the surface of a large sphere of radius $R$ determines the allowed wave numbers $k_{n}{ }^{0}$ according to $k_{n}{ }^{0} R-(l \pi / 2)=n \pi$ and thus $\Delta \epsilon_{n l}=\pi k_{n}{ }^{0} /\left(m_{r} R\right)$ is the spacing between, say, the $n$th and $(n+1)$ st level $(m$. is the reduced mass of the two-particle system). With interaction we have the wave numbers related to the phase shift of the asymptotic wave function $\delta_{l}$ by $k_{n} R-$ $(l \pi) / 2+\delta_{l}=n \pi$. Then

$$
\Delta E_{n l} \cong \frac{k_{n}{ }^{0}\left(k_{n}-k_{n}{ }^{0}\right)}{m_{r}}=-\frac{k_{n}{ }^{0}}{m_{r}} \frac{\delta_{s}\left(\epsilon_{n l}\right)}{R}
$$

$$
=-\frac{\delta_{l}\left(\epsilon_{n l}\right)}{\pi} \Delta \epsilon_{n l} .
$$

Evidently in the limit as $R \rightarrow \infty, \Delta \epsilon_{n l} \rightarrow 0$ and the sum over $n$ in Eq. (5) becomes an integral and we have in this same limit

$$
f_{l}(E)=-\frac{\operatorname{Im}}{\pi} \int_{0}^{\infty} d \epsilon \frac{\delta_{l}(\epsilon)}{E+i \eta-\epsilon}=\delta_{l}(E) .
$$

Let us write now the result of the preceding considerations as $\operatorname{Im} \ln D_{l_{+}}(E)=-\Delta_{l}(E)$ with

$$
\Delta_{l}(E)=\pi \sum_{B} \theta\left(E-E_{B}{ }^{(l)}\right) \theta(-E)+\theta\left(E^{l}\right) \delta_{l}(E),
$$

where in order that $\Delta_{l}(E)$ be continuous at $E=0$, we note that $\delta_{l}(0)=N^{(l)} \pi$ where $N^{(l)}$ is the total number of bound states of angular momentum $l$. We note that

$$
\frac{\left[D_{l+}(E)\right]^{*}}{D_{l+}(E)}=e^{2 i \Delta_{l}(E)},
$$

which has a very suggestive form: for $E>0$, this is just the $S$ matrix describing the scattering at energy $E$ in the particular angular momentum state. We shall return to this point later.

The evaluation of Eq. (2) may now be effected trivially. We write

$$
\begin{aligned}
z=\frac{1}{2 \pi i} \int_{B_{L}}^{\infty} d E e^{-\beta E} \frac{d}{d E} \ln \frac{\left[D_{+}(E)\right]^{*}}{D_{+}(E)} \\
=-\frac{1}{\pi} \int_{E_{L}}^{\infty} d E e^{-\beta E} \frac{d}{d E} \operatorname{Im} \ln D_{+}(E),
\end{aligned}
$$

where $E_{L}(<0)$ is less than any of the bound state energies. By substituting $D=\Pi\left[D_{l}\right]^{2 t+1}$ and using Eq. (7) we have

$$
\begin{aligned}
z= & \frac{1}{\pi} \int_{B_{L}}^{\infty} d E e^{-\beta E} \sum_{l}(2 l+1) \\
& \cdot\left[\pi \sum_{B} \delta\left(E-E_{B}{ }^{(l)}\right)+\theta(E) \frac{d \delta_{l}(E)}{d E}\right] \\
= & \sum_{B} \sum_{l}(2 l+1) \exp \left[-\beta E_{B}{ }^{(l)}\right] \\
& +\frac{1}{\pi} \int_{0}^{\infty} d E e^{-\beta E} \sum_{l}(2 l+1) \frac{d \delta_{l}(E)}{d E},
\end{aligned}
$$

which is the correct answer. ${ }^{1}$ It is important to note that if one integrates by parts in Eq. (10), the fact that $\delta(0)=N^{(l)} \pi$ must be taken into account.

These results may be generalized readily to the case where the potential is not spherically symmetric, so that the separation into angular moments is not possible. One needs simply replace the phase shifts $\delta_{l}(E)$ by the eigenphase shifts of the $S$ matrix, $\delta_{\lambda}(E)$. These are the phase shifts in the states which diagonalize the $S$ matrix. We write now

$$
\begin{array}{r}
\frac{\left[D_{+}(E)\right]^{*}}{D_{+}(E)}=\exp 2 i\left\{\sum_{\lambda} \sum_{B} \pi \theta\left(E-E_{B}{ }^{(\lambda)}\right)\right. \\
\left.\cdot \theta(-E)+\theta(E) \sum_{\lambda} \delta_{\lambda}(E)\right\}
\end{array}
$$


If we confine our attention from now on to $E>0$ we see that

$$
\frac{\left[D_{+}(E)\right]^{*}}{D_{+}(E)}=\exp 2 i \sum_{\lambda} \delta_{\lambda}(E)=\operatorname{det} S(E),
$$

where the identification made with the determinant of the $S$ matrix at energy $E$ is shown in detail by DeWitt. ${ }^{4}$ It is also evident from the fact that in its eigen-representation

$$
S(E)=\sum_{\lambda}\left|\lambda, E>e^{2 i b_{\lambda}(E)}<\lambda, E\right| .
$$

The expression of $D_{+}{ }^{*} / D_{+}$in terms of $\operatorname{det} S$ seems to be quite useful in connection with various approximation methods. Following DeWitt we write

$$
\begin{gathered}
\frac{1}{E_{+}-H_{0}}\left(E_{\perp}-H\right)=1-G_{0+}(E) V, \\
\begin{aligned}
G_{0+}(E) & \equiv \frac{1}{E_{+}-H_{0}}=\frac{P}{E-H_{0}}-i \pi \delta\left(E-H_{0}\right) \\
& \equiv \bar{G}_{0}-i \pi \delta\left(E-H_{0}\right),
\end{aligned}
\end{gathered}
$$

where the $P$ symbol means that in any integrations one takes the principle value. Introducing $G_{0-}=$ $G_{0+}{ }^{*}$, form a quantity $L(E)$

$$
L(E)=\left[1-G_{0-}\left(E^{\prime}\right) V\right] \frac{1}{1-G_{0+}(E) V} .
$$

It is evident by its construction that

$$
\operatorname{det} L(E)=D_{+}{ }^{*}(E) / D_{+}(E),
$$

and presumably then $\operatorname{det} L(E)=\operatorname{det} S(E)$. Next we write

$$
\begin{aligned}
L(E) & =1+\left(G_{0+}-G_{0-}\right) V \frac{1}{1-G_{0+} V} \\
& =1-2 \pi i \delta\left(E-H_{0}\right) V \frac{1}{1-G_{0+}} \bar{V}
\end{aligned}
$$

and it is from this form that DeWitt shows det $L(E)=\operatorname{det} S(E)$. It is important to note that the matrix elements of $L(E)$ are not equal to those of the $S$ matrix; only the determinants are equal. We proceed in a different manner and remark that

$$
L(E)=1-2 \pi i \frac{\delta\left(E-H_{0}\right) V \frac{1}{1-\bar{G}_{0} V}}{1+\pi i \delta\left(E-H_{0}\right) V \frac{1}{1-\bar{G}_{0} V}} .
$$

This may be easily verified by multiplying numerator and denominator by $1+G_{0} V$ from the right. The combination of operators which occurs in (18) is known in scattering theory as the reaction matrix.

- See, for example, B. A. Lippmann and J. Schwinger, Phys. Rev. 79, 469 (1950).
More precisely, it is defined as

$$
\begin{aligned}
K(E) & =V \frac{1}{1-\bar{G}_{0}(E) V} \\
& =V+G_{0}(E) V K(E),
\end{aligned}
$$

and in terms of this quantity

$$
L(E)=\frac{1-i \pi \delta\left(E-H_{0}\right) K(E)}{1+i \pi \delta\left(E-H_{0}\right) K(E)}
$$

We note also that the relevant (i.e., on the energy shells) matrix elements of $K$ may be expressed in terms of the standing wave solution of the Schroedinger equation $\psi_{\mathbf{k}}{ }^{(1)}(\mathbf{r})$ as

$$
\begin{aligned}
\delta\left(E_{\mathbf{k}^{\prime}}-E_{\mathbf{k}}\right) K_{\mathbf{k}^{\prime} \mathbf{k}}= & \delta\left(E_{\mathbf{k}^{\prime}}-E_{\mathbf{k}}\right) \\
& \cdot \int d^{3} r e^{-i \mathbf{k}^{\prime} \cdot \mathbf{r}} V(\mathbf{r}) \psi_{\mathbf{k}}^{(1)}(\mathbf{r}),
\end{aligned}
$$

and

$$
\psi_{\mathbf{k}}^{(1)}(\mathbf{r})=e^{i \mathbf{k} \cdot \mathbf{r}}+\frac{P}{E_{\mathbf{k}}-H_{0}} V \psi_{\mathbf{k}}^{(1)}(\mathbf{r})
$$

where $E_{\mathrm{k}}=\mathrm{k}^{2} / 2 m_{r}$. In the case of a spherically symmetric potential we have ${ }^{6}$ (with $\left|\mathbf{k}^{\prime}\right|=|\mathbf{k}|=k$ )

$K_{\mathbf{k}^{\prime} \mathbf{k}}=-\frac{4 \pi}{2 m_{r} k} \sum(2 l+1) \tan \delta_{l}(k) P_{l}\left(\mathbf{k}^{\prime} \cdot \mathbf{k}\right)$.

An approximation which naturally suggests itself is an expansion of $\operatorname{det} L(E)$ in powers of $K$. This may be easily done using the formula $\operatorname{det} A=$ exp \{trace In $A\}$. We have then immediately

$$
\text { det } \begin{aligned}
L(E) & =\operatorname{det} S(E) \\
\cong & \exp \left\{-\operatorname{trace} 2 \pi i \delta\left(E-H_{0}\right) K(E)\right\} \\
= & \exp \left\{-2 \pi i \int \frac{d^{3} k^{\prime}}{(2 \pi)^{3}} \delta\left(\frac{k^{\prime 2}}{2 m_{r}}-\frac{k^{2}}{2 m_{r}}\right)\right. \\
& \left.\cdot \int d^{3} r e^{-i \mathbf{k}^{\prime \cdot r}} V(\mathbf{r}) \psi_{\mathbf{k}}^{(1)}(\mathbf{r})\right\},
\end{aligned}
$$

where $E_{\mathbf{k}}$ has been written as $k^{2} / 2 m_{r}$. Using Eqs. (21) and (23) we have

$$
\operatorname{det} S(E) \cong \exp \left\{2 i \sum_{i}(2 l+1) \tan \delta_{i}(E)\right\}
$$

The next term in the expansion involves $(\tan \delta)^{3}$.

The first term in the expansion of $\ln \operatorname{det} S(E)$ in powers of the interaction is trivially obtained from Eq. (25) by the replacement of $\psi_{\mathbf{k}}{ }^{(1)}(\mathbf{r})$ by exp $(i \mathbf{k} \cdot \mathbf{r})$, in which case we have, carrying out the integration

$$
\operatorname{det} S(E) \cong \exp \left\{-2 i k \frac{m_{r}}{2 \pi} \int d^{3} r V(\mathbf{r})\right\}
$$


and we note that $-\left(m_{r} / 2 \pi\right) \int d^{3} r V(\mathbf{r})$ is just the Born approximation to the forward scattering amplitude. The expansion may, of course, be obtained directly from Eq. (15) as

$\operatorname{det} L(E)=\operatorname{det} S(E)$

$$
\begin{aligned}
\cong & \exp \left\{-\operatorname{trace} G_{0_{-}} V+\operatorname{trace} G_{0^{+}} V\right\} \\
= & \exp \operatorname{trace}\left\{\left[-\frac{1}{E-i \eta-H_{0}}\right.\right. \\
& \left.\left.+\frac{1}{E+i \eta-H_{0}}\right] V\right\} \\
= & \exp \operatorname{trace}\left\{-2 \pi i \delta\left(E-H_{0}\right) V\right\},
\end{aligned}
$$

which then leads directly to Eq. (27).
We plan to apply the methods described in this paper to certain many-body problems. One possible virtue of this approach is that it concentrates directly on what is needed, namely the phase of $D_{+}(E)$, and never calculates anything irrelevant. Furthermore, it seems to avoid a difficulty found in several other approximation techniques ${ }^{7}$ which show an alarming tendency to yield as a presumably exact result in the low-density region something equivalent to Eq. (26). Methods have been devised to avoid this but they are rather involved. ${ }^{8}$

7 K. A. Brueckner, Phys. Rev. 97, 1353 (1955); N. Hugenholtz (unpublished lecture, Princeton, New Jersey, December, 1958).

${ }^{8}$ W. Riesenfeld and K. M. Watson, Phys. Rev. 104, 492 (1956). 